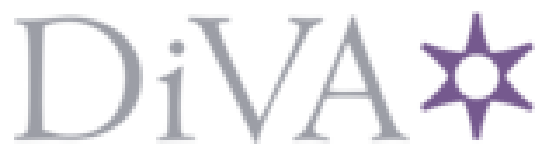

http://www.diva-portal.org

This is the published version of a paper published in .

Citation for the original published paper (version of record):

Bittencourt, A C., Wernholt, E., Sander Tavallaey, S., Brog?rdh, T. (2010)

An extended friction model to capture load and temperature effects in robot joints IEEE/RSJ 2010 International Conference on Intelligent Robots and Systems, IROS 2010 - Conference Proceedings, : 6161-6167

Access to the published version may require subscription.

N.B. When citing this work, cite the original published paper.

Permanent link to this version:

http://urn.kb.se/resolve?urn=urn:nbn:se:kth:diva-298582 


\title{
An Extended Friction Model to capture Load and Temperature effects in Robot Joints
}

\author{
André Carvalho Bittencourt, Erik Wernholt, Shiva Sander-Tavallaey and Torgny Brogårdh
}

\begin{abstract}
Friction is the result of complex interactions between contacting surfaces in a nanoscale perspective. Depending on the application, the different models available are more or less suitable. Available static friction models are typically considered to be dependent only on relative speed of interacting surfaces. However, it is known that friction can be affected by other factors than speed.

In this paper, static friction in robot joints is studied with respect to changes in joint angle, load torque and temperature. The effects of these variables are analyzed by means of experiments on a standard industrial robot. Justified by their significance, load torque and temperature are included in an extended static friction model. The proposed model is validated in a wide operating range, reducing the average error a factor of 6 when compared to a standard static friction model.
\end{abstract}

\section{INTRODUCTION}

Friction exists in all mechanisms to some extent. It can be defined as the tangential reaction force between two surfaces in contact. It is a nonlinear phenomenon which is physically dependent on contact geometry, topology, properties of the materials, relative velocity, lubricant, etc. [1]. Friction has been constantly investigated by researchers due to its importance in several fields [2]. In this paper, friction has been studied based on experiments on an industrial robot.

One reason for the interest in friction of manipulator joints is the need to model friction for control purposes [3]-[7], where a precise friction model can considerably improve the overall performance of a manipulator with respect to accuracy and control stability. Since friction can relate to the wear down process of mechanical systems [8], including robot joints [9], there is also interest in friction modeling for robot condition monitoring and fault detection [9]-[16].

A friction model consistent with real experiments is necessary for successful simulation, design and evaluation. Due to the complexity of friction, it is however often difficult to obtain models that can describe all the empirical observations (see [1] for a comprehensive discussion on friction physics and first principle friction modeling). In a robot joint, the complex interaction of components such as gears, bearings and shafts which are rotating/sliding at different velocities, makes physical modeling difficult. An example of an approach to model friction of complex transmissions can be

This work was supported by ABB and the Vinnova Industry Excellence Center LINK-SIC at Linköping University.

A. C. Bittencourt and E. Wernholt are with the Division of Automatic Control, Department of Electrical Engineering, Linköping University, Linköping, Sweden [andrecb, erikw] disy.liu.se

S. Sander-Tavallaey is with ABB Corporate Research, Västerås, Sweden shiva.sander-tavallaeylse.abb.com

T. Brogårdh is with ABB Robotics, Västerås, Sweden torgny.brogardhese.abb.com found in [17], where the author designs joint friction models based on physical models of elementary joint components as helical gear pairs and pre-stressed roller bearings.

Empirically motivated friction models have been successfully used in many applications, including robotics [5], [18] [20]. This category of models was developed through time according to empirical observations of the phenomenon [2]. Considering a set of states, $\mathcal{X}$, and parameters, $\theta$, these models can be described as the sum of $N$ functions $f_{i}$ that describe the behavior of friction, $\mathcal{F}$,

$$
\mathcal{F}(\mathcal{X}, \theta)=\sum_{i=1}^{N} f_{i}(\mathcal{X}, \theta) .
$$

The choice $\mathcal{X}=[z, \dot{q}, q]$, where $z$ is an internal state related to the dynamic behavior of friction, $q$ is a generalized coordinate and $\dot{q}=d q / d t$, gives the set of Generalized empirical Friction Model structures (GFM) [1].

Among the GFM model structures, the LuGre model [5], [19] is a common choice in the robotics community. For a revolute joint, it can be described as

$$
\begin{aligned}
\tau_{f} & =\sigma_{0} z+\sigma_{1} \dot{z}+h\left(\dot{\varphi}_{m}\right) \\
\dot{z} & =\dot{\varphi}_{m}-\sigma_{0} \frac{\left|\dot{\varphi}_{m}\right|}{g\left(\dot{\varphi}_{m}\right)} z,
\end{aligned}
$$

where $\tau_{f}$ is the friction torque and $\varphi_{m}$ is the joint motor angle. The state $z$ is related to the dynamic behavior of asperities in the interacting surfaces and can be interpreted as their average deflection, with stiffness $\sigma_{0}$ and damping $\sigma_{1}$. The function $h\left(\dot{\varphi}_{m}\right)$ represents the velocity strengthening (viscous) friction, typically taken as $h\left(\dot{\varphi}_{m}\right)=F_{v} \dot{\varphi}_{m}$, and $g\left(\dot{\varphi}_{m}\right)$ captures the velocity weakening of friction. Motivated by the observations of Stribeck [18], [21], $g\left(\dot{\varphi}_{m}\right)$ is usually modeled as

$$
g(\dot{\varphi})=F_{c}+F_{s} e^{-\left|\frac{\dot{\varphi}_{m}}{\dot{\varphi}_{s}}\right|^{\alpha}} .
$$

Where $F_{c}$ is the Coulomb friction, $F_{s}$ is defined as the standstill friction parameter $^{\dagger}, \dot{\varphi}_{s}$ is the Stribeck velocity and $\alpha$ is the exponent of the Stribeck nonlinearity. The model structure $\mathcal{M}_{L}$ is a GFM with $\mathcal{X}=\left[z, \dot{\varphi}_{m}\right]$ and $\theta=\left[\sigma_{0}, \sigma_{1}, F_{c}, F_{s}, F_{v}, \dot{\varphi}_{s}, \alpha\right]$. According to [19] it can successfully describe many of the friction characteristics.

Since $z$ is not measurable, a difficulty with $\mathcal{M}_{L}$ is the estimation of the dynamic parameters $\left[\sigma_{0}, \sigma_{1}\right]$. In [5], these parameters are estimated in a robot joint by means of open

\footnotetext{
${ }^{\dagger} F_{s}$ is commonly called static friction. An alternative nomenclature was adopted to make a distinction between the dynamic/static friction phenomena.
} 
loop experiments and by use of high resolution encoders. Open-loop experiments are not always possible, and it is common to accept only a static description of $\mathcal{M}_{L}$. For constant velocities, $\mathcal{M}_{L}$ is equivalent to the static model $\mathcal{M}_{S}$ :

$$
\tau_{f}(\dot{\varphi})=g\left(\dot{\varphi}_{m}\right) \operatorname{sign}\left(\dot{\varphi}_{m}\right)+h\left(\dot{\varphi}_{m}\right)
$$

which is fully described by the $g$ - and $h$ functions. In fact, $\mathcal{M}_{L}$ simply adds dynamics to $\mathcal{M}_{S}$. The typical choice for $g$ and $h$, as defined previously for $\mathcal{M}_{L}$, yields the static model structure $\mathcal{M}_{0}$ :

$$
\tau_{f}\left(\dot{\varphi}_{m}\right)=\left[F_{c}+F_{s} e^{-\left|\frac{\dot{\varphi}_{m}}{\dot{\varphi}_{s}}\right|^{\alpha}}\right] \operatorname{sign}\left(\dot{\varphi}_{m}\right)+F_{v} \dot{\varphi}_{m} .
$$

$\mathcal{M}_{0}$ requires a total of $4^{\dagger}$ parameters to describe the velocity weakening regime $g\left(\dot{\varphi}_{m}\right)$ and 1 parameter to capture viscous friction $h\left(\dot{\varphi}_{m}\right)$. See Fig. 3 for an interpretation of the parameters.

From empirical observations, it is known that friction can be affected by several factors,

- temperature,

- force/torque levels,

- position,

A shortcoming of the LuGre model structure, as with any GFM, is the dependence only of the states $\mathcal{X}=[z, \dot{q}, q]$. In more demanding applications, the effects of the remaining variables can not be neglected. In [17], the author observes a strong temperature dependence, while in [5] joint load torque and temperature are considered as disturbances and estimated in an adaptive framework. In [9], the influence of both joint load torque and temperature are observed. However, more work is needed in order to understand the influence of different factors on the friction properties. A more comprehensive friction model is needed to improve tasks related to design, simulation and evaluation for machines with friction.

The objective of this paper is to analyze and model the effects in static friction related to joint angle, load torques and temperature. The phenomena are observed in joint 2 of an ABB IRB 6620 industrial robot, see Fig. 1(a). Two load torque components are examined, the torque aligned to the joint $D o F$ (degree of freedom) and the torque perpendicular to the joint $D o F$. These torques are in the paper named manipulation torque $\tau_{m}$ and perpendicular torque $\tau_{p}$, see Fig. 1(b).

By means of experiments, these variables are analyzed and modeled based on the empirical observations. The task of modeling is to find a suitable model structure according to:

$$
\begin{aligned}
\tau_{f}\left(\mathcal{X}^{*}, \theta\right) & =\sum_{i=1}^{N} f_{i}\left(\mathcal{X}^{*}, \theta\right) \\
\mathcal{X}^{*} & =\left[\dot{\varphi}_{m}, \varphi_{a}, \tau_{p}, \tau_{m}, T\right],
\end{aligned}
$$

where $T$ is the joint (more precisely, lubricant) temperature and $\varphi_{a}$ the joint angle at arm side.

\footnotetext{
${ }^{\dagger}$ Many times $\alpha$ is considered a constant between 0.5 and 2 [19].
}

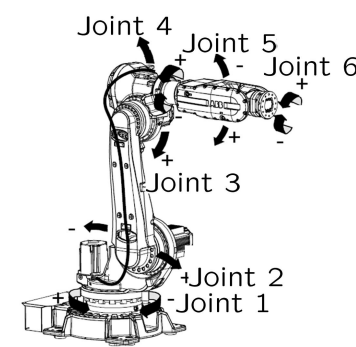

(a) ABB IRB 6620 robot with $150 \mathrm{~kg}$ payload and $2.2 \mathrm{~m}$ reach.

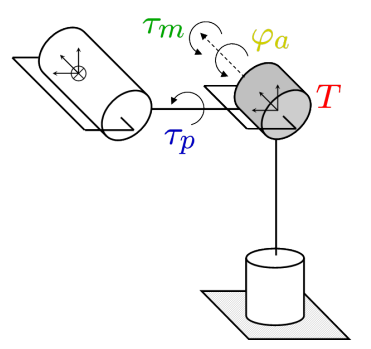

(b) Schematics of the 3 first joints including the torque definitions for joint 2 .
Fig. 1. The experiments were made on joint 2 of the ABB robot IRB 6620. $\varphi_{a}$ is the joint angle, $T$ the joint temperature, $\tau_{m}$ the manipulation torque and $\tau_{p}$ the perpendicular torque.

Ideally, the chosen model should be coherent with the empirical observations and, simultaneously, with the lowest dimension of $\theta$, the parameter vector, and with the lowest number of describing functions (minimum $N$ ). For practical purposes, the choice of $f_{i}$ should also be suitable for a useful identification procedure.

The paper is organized as follows. Section II presents the method used to estimate static friction in a robot joint, together with the guidelines used during the experiments. Section III contains the major contribution of this paper, with the empirical analysis, modeling and validation. Conclusions and future work are presented in Section IV.

\section{STATIC FRICTION ESTIMATION AND EXPERIMENTATION}

A manipulator is a multivariable, nonlinear system that can be described in a general manner through the rigid body dynamic model

$$
M\left(\varphi_{a}\right) \ddot{\varphi}_{a}+C\left(\varphi_{a}, \dot{\varphi}_{a}\right)+\tau_{g}\left(\varphi_{a}\right)+\tau_{f}=u
$$

where $\varphi_{a}$ and $\varphi_{m}^{\ddagger}$ are the vectors of robot angles at arm and motor side of the joint gearbox, $M\left(\varphi_{a}\right)$ is the inertia matrix, $C\left(\varphi_{a}, \dot{\varphi}_{a}\right)$ relates to speed dependent terms (e.g. Coriolis and centrifugal), $\tau_{g}\left(\varphi_{a}\right)$ are the gravity-induced torques and $\tau_{f}$ contain the joint friction components. The system is controlled through the input torque, $u$, applied to the joint motor (in the experiments the torque reference from the servo was measured ${ }^{\S}$ ).

For single joint movements $\left(C\left(\varphi_{a}, \dot{\varphi}_{a}\right)=0\right.$ at that joint $)$ under constant speed ( $\ddot{\varphi}_{a} \approx 0$ ), Equation (1) simplifies to

$$
\tau_{g}\left(\varphi_{a}\right)+\tau_{f}=u
$$

The applied torque $u$ drives only friction and gravity-induced torques. The required torques to drive a joint in forward, $u^{+}$, and reverse, $u^{-}$, directions at constant speed $\bar{\varphi}_{m}$ and at a

\footnotetext{
${ }^{\ddagger}$ Notice that for the rigid model (1) follows the equivalence $\varphi_{a}=r \cdot \varphi_{m}$, where $r$ is the gearbox ratio. Both nomenclatures are kept to emphasize friction as a joint phenomenon.

$\S$ It is known that this abstraction might not always hold, for instance under high temperatures. The deviations are however expected to be small and therefore neglected during the experiments.
} 
joint angle $\bar{\varphi}_{a}$ (so that $\tau_{g}\left(\varphi_{a}\right)$ is equal in both directions), are

$$
\begin{aligned}
\tau_{f}\left(\overline{\dot{\varphi}}_{m}\right)+\tau_{g}\left(\bar{\varphi}_{a}\right) & =u^{+} \\
\tau_{f}\left(-\dot{\bar{\varphi}}_{m}\right)+\tau_{g}\left(\bar{\varphi}_{a}\right) & =u^{-} .
\end{aligned}
$$

In case an estimate of $\tau_{g}\left(\bar{\varphi}_{a}\right)$ is available, it is simple to isolate the friction estimates for both directions. If such estimate is not possible (e.g. not all masses are completely known), $\tau_{f}$ can still be estimated as follows. Subtracting the equations yields

$$
\tau_{f}\left(\overline{\dot{\varphi}}_{m}\right)-\tau_{f}\left(-\overline{\dot{\varphi}}_{m}\right)=u^{+}-u^{-}
$$

and supposing a direction independent friction, $\tau_{f}\left(-\bar{\varphi}_{m}\right)=-\tau_{f}\left(\overline{\dot{\varphi}}_{m}\right)$, the resulting direction independent friction is:

$$
\tau_{f}\left(\overline{\dot{\varphi}}_{m}\right)=\frac{u^{+}-u^{-}}{2} .
$$

Due to nonlinearities of friction, it is important to define an excitation signal including several different (constant) velocities. The signal used moves one axis at a time at 12 speed levels in both directions, taking 2:15 min and sampled at $2 \mathrm{KHz}^{\dagger}$. Fig. 2 shows the motor speed- and torque ${ }^{\ddagger}$ signals in the experiments.

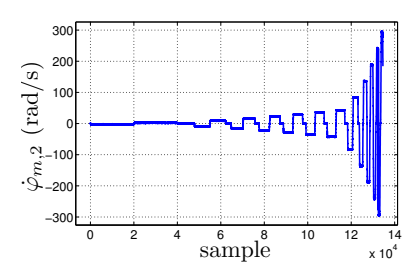

(a) Motor speed

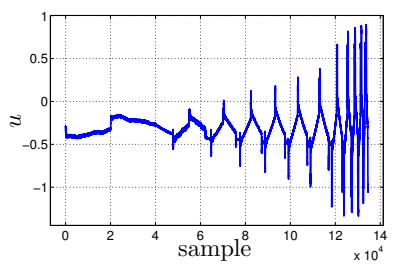

(b) Motor applied torque
Fig. 2. Excitation signal used for the static friction curve estimation.

The data was segmented at the different constant speeds and friction estimates are obtained using (3) and (4). The result of the estimation can then be presented in a static friction curve, sometimes reffered as Stribeck curve, see Fig. 3. Motivated by the small direction dependency of friction for this joint, the estimation approach considered further in this work is based in Equation (4), which has better sensitivity to noise due to the averaging.

\section{A. Parametric Description and Identification}

The static models considered throughout this work can be written as a pseudolinear regression [22],

$$
\hat{\tau}_{f}\left(\dot{\varphi}_{m}, \theta\right)=\mathbf{f}\left(\dot{\varphi}_{m}, \theta_{2}\right) \theta_{1}^{T}
$$

where $\mathbf{f}\left(\dot{\varphi}_{m}, \theta_{2}\right)$ is a regressor vector. The parameters vector $\theta=\left[\theta_{1}, \theta_{2}\right]$ is divided according to the manner they appear in the model, respectively linearly/nonlinearly. Notice that

\footnotetext{
${ }^{\dagger}$ Similar results have been experienced with sampling rates down to $220 \mathrm{~Hz}$.

${ }^{\ddagger}$ Throughout the paper all torques are normalized to the maximum manipulated torque at low speed.
}

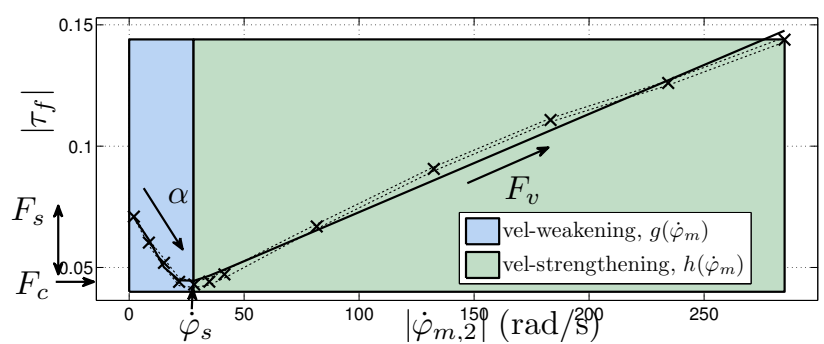

Fig. 3. Estimated static friction curve and the $\mathcal{M}_{0}$ parametric representation. The solid line presents the $\mathcal{M}_{0}$ predictions for the parameters obtained by best fit with the data presented as crosses. Crosses, friction torque values estimated using Eq. (4), with the assumption that friction is direction independent; dotted lines, friction torques estimated using Eq. (3).

if $\theta_{2}$ was fixed, $\theta_{1}$ could be estimated with a simple linear regression. The chosen identification method combines linear regression for $\theta_{1}$ with extensive search (grid search over a predetermined range) for $\theta_{2}$. The parameters yielding the smallest absolute sum prediction of errors, $\varepsilon$, is then chosen,

$$
\hat{\theta}=\arg \min _{\theta} \sum|\varepsilon|=\arg \min _{\theta} \sum\left|\tau_{f}-\hat{\tau}_{f}\left(\dot{\varphi}_{m}, \theta\right)\right| .
$$

For the model structure $\mathcal{M}_{0}$, Equation (5) can be written as

$$
\begin{array}{r}
\mathbf{f}\left(\dot{\varphi}_{m}, \theta_{2}\right)=\left[\mathbf{1}, e^{-\left|\frac{\dot{\varphi}_{m}}{\dot{\varphi}}\right|^{\alpha}}, \dot{\varphi}_{m}\right] \\
\theta_{1}=\left[F_{c}, F_{s}, F_{v}\right], \quad \theta_{2}=\left[\dot{\varphi}_{s}, \alpha\right] .
\end{array}
$$

The model parameters are identified using the direction independent data (crosses) in Fig. 3. The resulting identified parameters values are $\left[F_{c}, F_{s}, F_{v}, \dot{\varphi}_{s}, \alpha\right]=$ $\left[3.2410^{-2}, 3.8410^{-2}, 4.0410^{-4}, 13.80,1.91\right]$. The solid line in Fig. 3 is obtained by model-based predictions of the resulting model. Notice that the model structure $\mathcal{M}_{0}$ can satisfactorily describe static friction dependence on speed. In fact, the sum of absolute prediction errors in this case is no more than 0.03 .

\section{B. Guidelines for the Experiments}

In order to be able to build a friction model including more variables than the velocity, it is important to separate their influences. The situation is particularly critical regarding temperature as it is difficult to control it inside a joint. Moreover, due to the complex structure of an industrial robot, changes in joint angle might move the mass center of the robot arm system, causing variations of joint load torques. To avoid undesired effects, the guidelines below were followed during the experiments.

1) Isolating joint load torque dependency from joint angle dependency: Using an accurate dynamic robot model ${ }^{\S}$, it is possible to predict the joint torques for any given robot configuration (a set of all joints angles). For example, Fig. 4 shows the resulting $\tau_{m}$ and $\tau_{p}$ at joint 2 , related to variations of joint 2 and 4 angles $\left(\varphi_{a, 2}\right.$ and $\left.\varphi_{a, 4}\right)$ throughout their workrange. Using this information, a set of configurations can be selected a priori in which it is possible to estimate parameters in an efficient way.

\footnotetext{
$\S_{\text {An }}$ ABB internal tool was used for simulation purposes.
} 


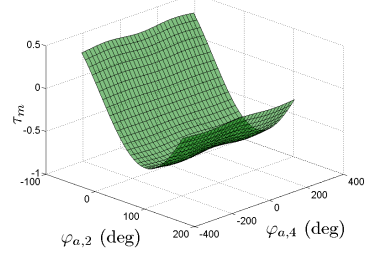

(a) Simulated $\tau_{m}$

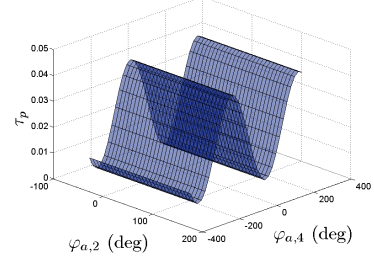

(b) Simulated $\tau_{p}$
Fig. 4. Simulated joint load torques at joint 2. Notice the larger absolute values for $\tau_{m}$ when compared $\tau_{p}$.

2) Isolating temperature effects: Some of the experiments require that the temperature of the joint is under control. Using joint lubricant temperature measurements ${ }^{\dagger}$, the joint thermal decay constant $\kappa$ was estimated to 3.04 h. Executing the static friction curve identification experiment periodically, for longer time than $2 \kappa$ (i.e. $>6.08 \mathrm{~h}$ ), the joint temperature is expected to have reached an equilibrium. Only data related to the expected thermal equilibrium was considered for the analysis.

\section{EMPIRICALLY MOTIVATED MODELING}

Using the described static friction curve estimation method, it is possible to design a set of experiments to analyze how the states $\mathcal{X}^{*}$ affect static friction. As shown in Section II-A, the model structure $\mathcal{M}_{0}$ can represent static friction dependence on $\dot{\varphi}_{m}$ fairly well. $\mathcal{M}_{0}$ is therefore taken as a primary choice. Since $\dot{\varphi}_{s}$ and $\alpha$ influence the resulting friction in a similar manner, $\alpha$ is typically considered fixed [5], [7], [19]. In this work, $\alpha$ is chosen as $1.3^{\ddagger}$. Whenever $\mathcal{M}_{0}$ can not describe the observed friction behavior, extra terms $f_{i}\left(\mathcal{X}^{*}, \theta\right)$ are proposed and included in $\mathcal{M}_{0}$ to achieve a satisfactory model structure $\mathcal{M}^{*}$.

\section{A. Joint angles}

Due to asymmetries in the contact surfaces, it has been observed that the friction of rotating machines depends on the angular position [1]. It is therefore expected that this dependency occurs also in a robot joint. Following the experiment guidelines from the previous section, a total of 50 static friction curves are estimated in the joint angle range $\varphi_{a}=[8.40,59.00] \mathrm{deg}$. As seen in Fig. 5(a), little effects can be observed. The subtle deviations are comparable to the errors of the friction curve identified under constant values of $\left[\varphi_{a}, \tau_{p}, \tau_{m}, T\right]$. In fact, even a constant instance of $\mathcal{M}_{0}$ can describe the friction curves satisfactorily, no extra $f_{i}$ terms are thus required.

\footnotetext{
$\dagger$ In the studies, the robot gearbox was lubricated with oil, not grease, which gave an opportunity to obtain well defined temperature readings by having a temperature sensor in the circulating lubricant oil.

${ }^{\ddagger}$ Considering all static friction data presented in this work, $\alpha=1.3$ minimizes Equation (6) for the model structure $\mathcal{M}_{0}$ when all other parameters are free.
}
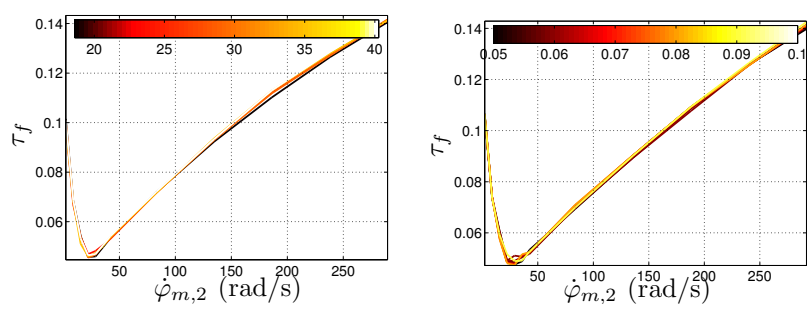

(a) Effects of $\varphi_{a}$ at $\tau_{m}=-0.39$, (b) Effects of $\tau_{p}$ at $\tau_{m}=-0.39$, $T=34^{\circ} \mathrm{C}$. $T=36^{\circ} \mathrm{C}$.

Fig. 5. Static friction curves for experiments related to $\varphi_{a}$ and $\tau_{p}$.

\section{B. Joint load torque}

Since friction is related to the interaction between contacting surfaces, one of the first phenomena observed was that friction varies according to the applied normal force. The observation is thought to be caused by the increase of the true contact area between the surfaces under larger normal forces. A similar reasoning can be extended to joint torques in a robot revolute joint. Due to the elaborated joint gear- and bearing design it is also expected that torques in different directions will have different effects on the static friction curve $^{\S}$.

Because of the mechanical construction of the robot, only small variations of the perpendicular load torque, $\tau_{p}$, are possible to achieve for joint 2 (see Fig. 4(b)). A total of 20 experiments at constant temperature were performed for joint 2 , in the range $\tau_{p}=[0.04,0.10]$. As Fig. 5(b) shows, $\tau_{p}$ values in the obtained range did not play a significant role for the static friction curve. No extra terms are therefore needed for joint 2 and $\mathcal{M}_{0}$ is considered valid. The observation is true at least over a narrow $\tau_{p}$ interval.

As seen in Fig. 4(a), large variations of the manipulation torque $\tau_{m}$ are possible by simply varying the arm configuration. A total of 50 static friction curves were estimated over the range $\tau_{m}=[-0.73,0.44]$. As seen in Fig. 6, the effects appear clearly. Obviously, a single $\mathcal{M}_{0}$ instance can not describe the observed phenomena. A careful analysis of the effects reveals that the main changes occur in the velocity weakening part of the curve. From Fig. 6(c), it is possible to observe a (linear) bias-like $\left(F_{c}\right)$ increase and a (linear) increase of the standstill friction $\left(F_{s}\right)$ with $\left|\tau_{m}\right|$. Furthermore, as seen in Fig. 6(b), the Stribeck velocity $\dot{\varphi}_{m}$ is maintained fairly constant. The observations support an extension of $\mathcal{M}_{0}$ to

$$
\begin{aligned}
& \tau_{f}\left(\dot{\varphi}_{m}, \tau_{m}\right)=\left\{F_{c, 0}+F_{c, \tau_{m}}\left|\tau_{m}\right|\right\}+ \\
& \quad+\left\{F_{s, 0}+F_{s, \tau_{m}}\left|\tau_{m}\right|\right\} e^{-\left|\frac{\dot{\varphi}_{m}}{\dot{\varphi}_{s, \tau_{m}}}\right|^{1.3}}+F_{v} \dot{\varphi}_{m}
\end{aligned}
$$

In the above equation the parameters are written with subscript ${ }_{-0}$ or ${ }_{-\tau_{m}}$ in order to clarify its origin related $\mathcal{M}_{0}$ or to the effects of $\tau_{m}$. Assuming that any phenomenon not related to $\tau_{m}$ is constant and such that the ${ }_{-0}$ terms can capture

\footnotetext{
$\S$ In fact, a full joint load description would require 3 torque and 3 force components.
} 


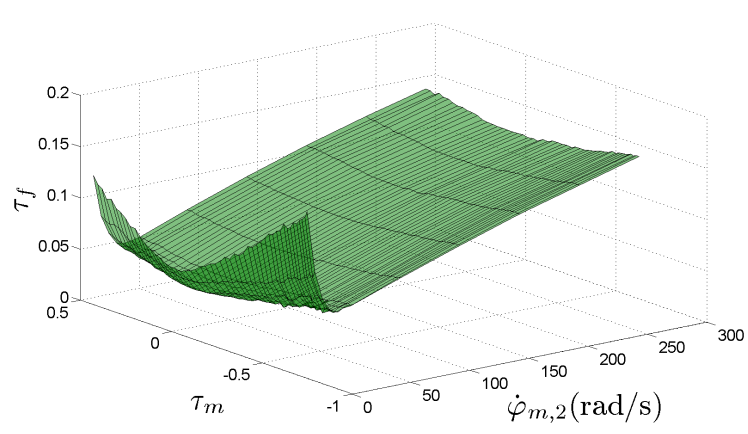

(a) Estimated friction curves for different values of $\tau_{m}$.

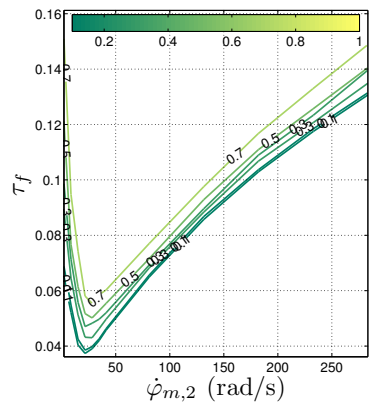

(b) Friction surface cuts for different values of $\tau_{m}$

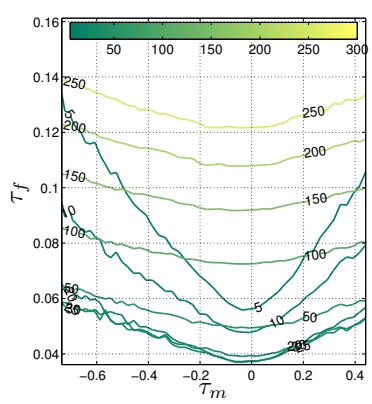

(c) Friction surface cuts for different values of $\dot{\varphi}_{m, 2}(\mathrm{rad} / \mathrm{s})$
Fig. 6. The dependence of the static friction curves on the manipulation torque, $\tau_{m}$, at $T=34^{\circ} \mathrm{C}$.

them, good estimates of the $\tau_{m}$-dependent parameters can be achieved. The model $\mathcal{M}_{1}$ is identified with the data set from Fig. 6 using the procedure illustrated in Section II-A, linear regression combined with grid search for $\dot{\varphi}_{s, \tau_{m}}$. The resulting model parameters describing the dependence on $\tau_{m}$ are shown in Table I.

TABLE I

IDENTIFIED $\tau_{m}$-DEPENDENT MODEL PARAMETERS.

\begin{tabular}{c|c|c}
\hline$F_{c, \tau_{m}}$ & $F_{s, \tau_{m}}$ & $\dot{\varphi}_{s, \tau_{m}}$ \\
\hline $2.3210^{-2}$ & $1.2810^{-1}$ & 9.07 \\
\hline
\end{tabular}

\section{Temperature}

The friction temperature dependence is related to the change of properties of both lubricant and contacting surfaces. In lubricated mechanisms, both the thickness of the lubricant layer and its viscosity play an important role for the resulting friction properties. In newtonian fluids, the shear forces are directly proportional to the viscosity which, in turn, varies with temperature [23]. Dedicated experiments were made to analyze temperature effects. The joint was at first warmed up to $81.2^{\circ} \mathrm{C}$ by running the joint continuously back and forth. Then, while the robot cooled, 50 static friction curves were estimated over the range $T=[38.00,81.20]^{\circ} \mathrm{C}$. In order to resolve combined effects of $T$ and $\tau_{m}$, two manipulation torque levels were used, $\tau_{m}=-0.02$, and $\tau_{m}=-0.72$. As it can be seen in Fig. 7, the effects of $T$ are significant.

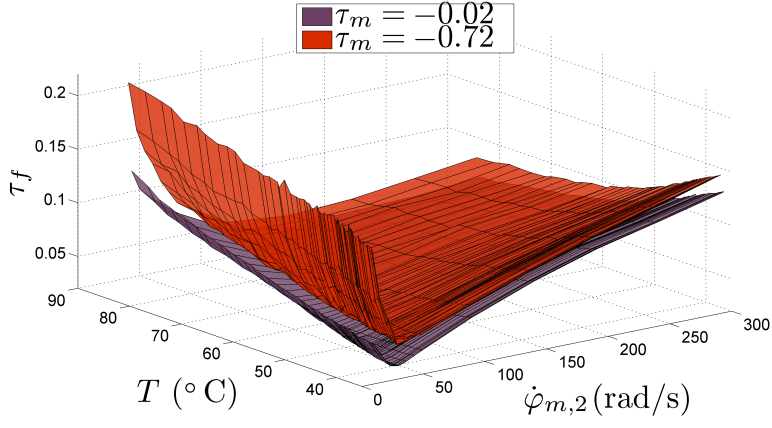

(a) Estimated friction curves for different values of $T$.

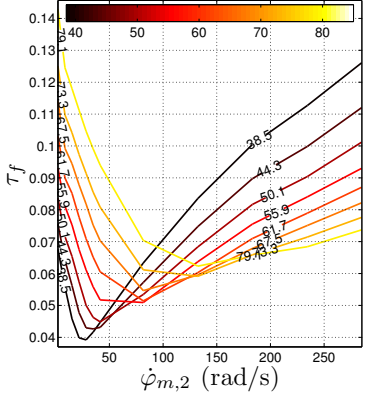
ent values of $T$ at $\tau_{m}=-0.02$. (b) Friction surface cuts for differ-

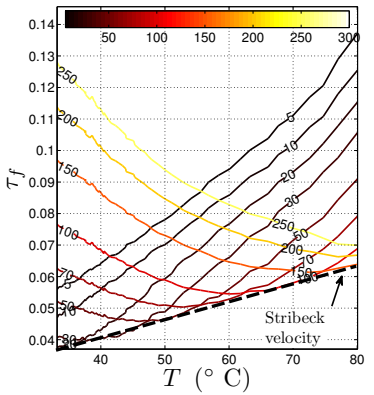

(c) Friction surface cuts for different values of $\dot{\varphi}_{m, 2}(\mathrm{rad} / \mathrm{s})$ at $\tau_{m}=-0.02$.
Fig. 7. The temperature dependence of the static friction curve.

Temperature has an influence on both velocity regions of the static friction curves. In the velocity-weakening region, a (linear) increase of the standstill friction $\left(F_{s}\right)$ with temperature can be observed according to Fig. 7(b). In Fig. 7(c) it can moreover be seen that the Stribeck velocity $\left(\dot{\varphi}_{s}\right)$ increases (linearly) with temperature. The effects in the velocitystrengthening region appear as a (nonlinear, exponential-like) decrease of the velocity-dependent slope, as seen in Fig. 7(b) and $7(\mathrm{c})$.

It is also interesting to study combined effects of $\tau_{m}$ and $T$. To better see these effects, the friction surfaces in Fig. 7(a) are subtracted from each other, yielding $\tilde{\tau}_{f}$. As it can be seen from the resulting surface in Fig. 8(a), the difference between the surfaces is fairly temperature independent. This is an indication of independence between effects caused by $T$ and $\tau_{m}$.

Given that the effects of $T$ and $\tau_{m}$ are independent, it is possible to subtract the $\tau_{m}$-effects from the surfaces in Fig. 7(a) and solely obtain temperature related phenomena. The previously proposed terms to describe the $\tau_{m}$-effects in $\mathcal{M}_{1}$ were:

$$
\hat{\tau}_{f}\left(\tau_{m}\right)=F_{c, \tau_{m}}\left|\tau_{m}\right|+F_{s, \tau_{m}}\left|\tau_{m}\right| e^{-\left|\frac{\dot{\varphi}_{m}}{\dot{\varphi}_{s, \tau_{m}}}\right|^{1.3}} .
$$

With the parameters values given from Table I, the manipulation torque effects were subtracted from the friction curves of the two surfaces in Fig. 7(a), that is, the quantities $\tau_{f}-\hat{\tau}_{f}\left(\tau_{m}\right)$ were computed. The resulting surfaces are shown in Fig. 8(b). As expected, the surfaces become quite similar. 
The result can also be interpreted as an evidence on the fact that the model structure used for the $\tau_{m}$-dependent terms and the identified parameter values are correct. Obviously,

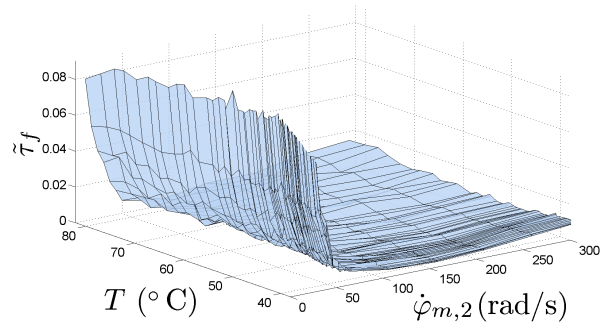

(a) Difference $\tilde{\tau}_{f}$ between the two static friction surfaces in Fig. 7(a).

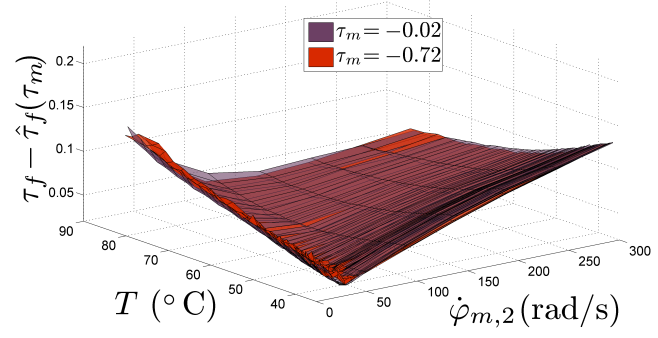

(b) Static friction surfaces in Fig. 7(a) after subtraction of the $\tau_{m}$-dependent terms.

Fig. 8. Indication of independence between effects caused by $T$ and $\tau_{m}$.

the original model structure $\mathcal{M}_{0}$ can not characterize all observed phenomena, even after discounting the $\tau_{m}$-dependent terms.

A proposal for $\mathcal{M}^{*}$. From the characteristics of the $T$ related effects and the already discussed $\tau_{m}$-effects, $\mathcal{M}_{1}$ is extended to:

$$
\begin{aligned}
& \tau_{f}\left(\dot{\varphi}_{m}, \tau_{m}, T\right)= \\
& \left\{F_{c, 0}+F_{c, \tau_{m}}\left|\tau_{m}\right|\right\}+F_{s, \tau_{m}}\left|\tau_{m}\right| e^{-\left|\frac{\dot{\varphi}_{m}}{\dot{\varphi}_{s, \tau_{m}}}\right|^{1.3}}+\left(\mathcal{M}_{g\left(\tau_{m}\right)}^{*}\right) \\
& +\left\{F_{s, 0}+F_{s, T} T\right\} e^{-\left|\frac{\dot{\varphi}_{m}}{\left\{\dot{\varphi}_{s, 0}+\dot{\varphi}_{s, T} T\right\}}\right|^{1.3}}+\quad\left(\mathcal{M}_{g(T)}^{*}\right) \\
& +\left\{F_{v, 0}+F_{v, T} e^{\frac{-T}{T_{\mathrm{Vo}}}}\right\} \dot{\varphi}_{m} . \\
& \left(\mathcal{M}_{h(T)}^{*}\right)
\end{aligned}
$$

The model describes the effects of $\tau_{m}$ and $T$ for the investigated robot joint. The first $\mathcal{M}_{g}^{*}$ expressions relate to the velocity-weakening friction while $\mathcal{M}_{h}^{*}$ relates to the velocity-strengthening regime. $\tau_{m}$ only affects the velocityweakening regime and requires a total of 3 parameters, $\left[F_{c, \tau_{m}}, F_{s, \tau_{m}}, \dot{\varphi}_{s, \tau_{m}}\right] . T$ affects both regimes and requires 4 parameters, $\left[F_{s, T}, \dot{\varphi}_{s, T}, F_{v, \tau_{m}}, T_{\mathrm{Vo}}\right]$. The 4 remaining parameters, $\left[F_{c, 0}, F_{s, 0}, \dot{\varphi}_{s, 0}, F_{v, 0}\right]$, relate to the original friction model structure $\mathcal{M}_{0}$. Notice that under the assumption that $\tau_{m}$ - and $T$ effects are independent, their respective expressions appear as separated sums in $\mathcal{M}^{*}$.

The term $F_{v, T} e^{-T / T_{\mathrm{Vo}}}$ in $\mathcal{M}_{h_{T}}^{*}$ is motivated by the exponential-like behavior of viscous friction (recall Fig. 7(c)). In fact, the parameter $T_{\mathrm{Vo}}$ is a reference to the Vogel-Fulcher-Tamman exponential description of viscosity

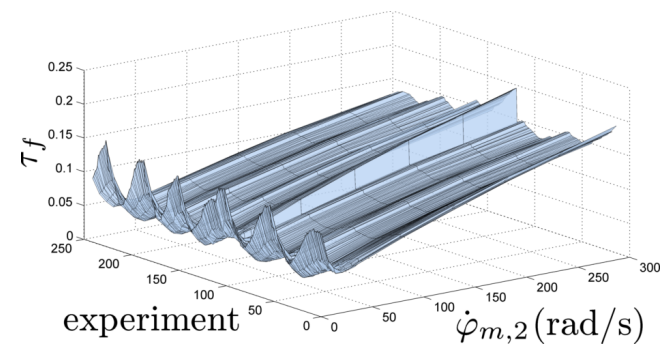

(a) Static friction curves

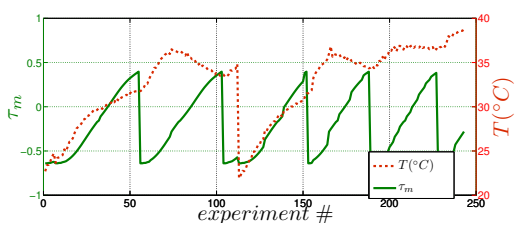

(b) $\tau_{m}$ - and $T$ conditions

Fig. 9. Validation data set. Notice the large variations of $T$ - and $\tau_{m}$ values in Fig. (b) when registering the static friction curves in (a).

and temperature [23]. Such behavior is observed in a large but limited temperature range, to capture the static friction behavior at even larger temperature ranges, more complex expressions may be needed, see [23] for other structures.

Given the already identified $\tau_{m}$-dependent parameters in Table I, the remaining parameters from $\mathcal{M}^{*}$ are identified from the measurement results presented in Fig. 8(b), after the subtraction of the $\tau_{m}$-terms. The values are shown in Table II.

\section{Validation}

A separate data set is used for the validation of the proposed model structure $\mathcal{M}^{*}$. It consists of several static friction curves measured at different $\tau_{m^{-}}$and $T$ values, as seen in Fig. 9. With an instance of $\mathcal{M}^{*}$ given by the parameter values from Tables I and II, the resulting prediction errors for the validation data set are shown in Fig. 10. As a comparison, the errors related to a single instance of $\mathcal{M}_{0}$, with $\left[F_{c}, F_{s}, F_{v}, \dot{\varphi}_{s}, \alpha\right]=$ $\left[4.9010^{-2}, 8.4410^{-2}, 5.0010^{-4}, 6.50,1.30\right]$, are also shown in the figure. As it can be seen, $\mathcal{M}^{*}$ performs visibly better

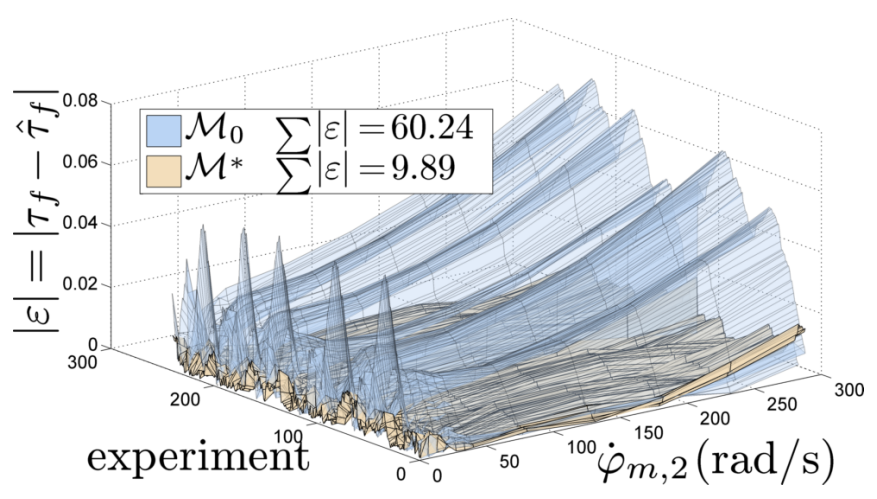

Fig. 10. Models absolute prediction error. Notice the considerable better performance of $\mathcal{M}^{*}$. 
TABLE II

IDENTIFIED $T$-DEPENDENT AND $\mathcal{M}_{0}$-RELATED MODEL PARAMETERS.

\begin{tabular}{c|c|c|c|c|c|c|c}
\hline$F_{c, 0}$ & $F_{s, 0}$ & $F_{s, T}$ & $F_{v, 0}$ & $F_{v, T}$ & $\dot{\varphi}_{s, 0}$ & $\dot{\varphi}_{s, T}$ & $T_{\mathrm{Vo}}$ \\
\hline $3.0410^{-2}$ & $-2.4410^{-2}$ & $1.6910^{-3}$ & $1.2910^{-4}$ & $1.3110^{-3}$ & -25.00 & 1.00 & 21.00 \\
\hline
\end{tabular}

when compared to $\mathcal{M}_{0}$, with only speed dependence. The maximum and mean errors for $\mathcal{M}^{*}$ are $\left[1.8610^{-2}, 3.3910^{-3}\right]$, compared to $\left[7.0910^{-2}, 2.0710^{-2}\right]$ for $\mathcal{M}_{0}$.

The proposed model structure has also been successfully validated in other joints with similar gearboxes, but it might be interesting to validate it in other robot types and even other types of rotating mechanisms.

\section{CONCLUSIONS AND FURTHER RESEARCH}

The main contribution of this paper is the empirically derived model of static friction as a function of the variables $\mathcal{X}^{*}=\left[\dot{\varphi}_{m}, \varphi_{a}, \tau_{p}, \tau_{m}, T\right]$. While no significant influences of joint angle and perpendicular torque could be found by the experiments, the effects of manipulation torque $\left(\tau_{m}\right)$ and temperature $(T)$ were significant and included in the proposed model structure $\mathcal{M}^{*}$. As shown in Fig. 10 the model is needed in applications where the manipulation torque and the temperature play significant roles.

In the studies, the friction phenomena was fairly direction independent, if this was not the case, two instances of $\mathcal{M}^{*}$ could be used to describe the whole speed range, but requiring two times more parameters. The model $\mathcal{M}^{*}$ has a total of 7 terms and 3 parameters which enter the model in a nonlinear fashion. The identification of such a model is computationally costly and requires data from several different operating conditions. Studies on defining sound identification excitation and estimation routines are therefore important.

Only static friction (measured when transients caused by velocity changes have disappeared) was considered in the studies. It would be interesting to investigate if a dynamic model, for instance given by the LuGre model structure $\mathcal{M}_{L}$, could be used to describe dynamic friction with extensions from the proposed $\mathcal{M}^{*}$. However, to make experiments on a robot joint in order to obtain a dynamic friction model is a big challenge. Probably, such experiments must be made on a robot joint mounted in a test bench instead of on a robot arm system, which has very complex dynamics.

A practical limitation of $\mathcal{M}^{*}$ is the requirement on availability of $\tau_{m}$ and $T$. Up to date, torque- and joint temperature sensors are not available in standard industrial robots. As mentioned in Section II-B, the joint torque components can still be estimated from the torque reference to the drive system by means of an accurate robot model. In this situation, it is important to have correct load parameters in the model to calculate the load torque components.

Regardless these experimental challenges, there is a great potential for the use of $\mathcal{M}^{*}$ for simulation-, design- and evaluation purposes. The designer of control algorithms, the diagnosis engineer, the gearbox manufacturer, etc. would benefit by using a more realistic friction model.

\section{REFERENCES}

[1] F. Al-Bender and J. Swevers, "Characterization of friction force dynamics," IEEE Control Systems Magazine, vol. 28, no. 6, pp. 64-81, 2008.

[2] D. Dowson, History of Tribology. Professional Engineering Publishing, London, UK., 1998.

[3] H. M. Kim, S. H. Park, and S. I. Han, "Precise friction control for the nonlinear friction system using the friction state observer and sliding mode control with recurrent fuzzy neural networks," Mechatronics, vol. 19 , no. 6, pp. $805-815,2009$.

[4] Y. Guo, Z. Qu, Y. Braiman, Z. Zhang, and J. Barhen, "Nanotribology and nanoscale friction," Control Systems Magazine, IEEE, vol. 28, no. 6, pp. $92-100$, dec. 2008.

[5] H. Olsson, K. J. Åström, C. C. de Wit, M. Gafvert, and P. Lischinsky, "Friction models and friction compensation," European Journal of Control, vol. 4, no. 3, pp. 176-195, 1998.

[6] B. Bona and M. Indri, "Friction compensation in robotics: an overview," in Decision and Control, 2005. Proceedings., 44th IEEE International Conference on, Dec 2005.

[7] F. L. Witono Susanto, Robert Babuska and T. van der Weiden, "Adaptive friction compensation: application to a robotic manipulator," in The International Federation of Automatic Control, 2008. Proceedings., 17th World Congress, Dec 2008.

[8] P. J. Blau, "Embedding wear models into friction models," Tribology Letters, vol. 34, no. 1, Apr. 2009.

[9] A. C. Bittencourt, "Friction change detection in industrial robot arms," MSc. thesis, The Royal Institute of Technology, 2007.

[10] F. Caccavale, P. Cilibrizzi, F. Pierri, and L. Villani, "Actuators fault diagnosis for robot manipulators with uncertain model," Control Engineering Practice, vol. 17, no. 1, pp. 146 - 157, 2009.

[11] M. Namvar and F. Aghili, "Failure detection and isolation in robotic manipulators using joint torque sensors," Robotica, 2009.

[12] M. McIntyre, W. Dixon, D. Dawson, and I. Walker, "Fault identification for robot manipulators," Robotics, IEEE Transactions on, vol. 21, no. 5, pp. 1028-1034, Oct. 2005.

[13] A. T. Vemuri and M. M. Polycarpou, "A methodology for fault diagnosis in robotic systems using neural networks," Robotica, vol. 22, no. 04, pp. 419-438, 2004.

[14] D. Brambilla, L. Capisani, A. Ferrara, and P. Pisu, "Fault detection for robot manipulators via second-order sliding modes," Industrial Electronics, IEEE Transactions on, vol. 55, no. 11, pp. 3954-3963, Nov. 2008.

[15] R. Mattone and A. D. Luca, "Relaxed fault detection and isolation: An application to a nonlinear case study," Automatica, vol. 42, no. 1, pp. $109-116,2009$.

[16] B. Freyermuth, "An approach to model based fault diagnosis of industrial robots," in Robotics and Automation, 1991. Proceedings., 1991 IEEE International Conference on, vol. 2, Apr 1991, pp. 13501356.

[17] R. Waiboer, "Dynamic modelling, identification and simulation of industrial robots," Ph.D. dissertation, University of Twente, 2007.

[18] B. Armstrong-Hélouvry, Control of Machines with Friction. Kluwer Academic Publishers, 1991.

[19] K. J. Åström and C. Canudas-de Wit, "Revisiting the lugre friction model," Control Systems Magazine, IEEE, vol. 28, no. 6, pp. 101114, Dec. 2008.

[20] B. F. S. C. Avraham Harnoy, "Modeling and measuring friction effects," Control Systems Magazine, IEEE, vol. 28, no. 6, Dec. 2008.

[21] R. Stribeck, "Die wesentlichen eigenschaften der gleit-und rollenlager - the key qualities of sliding in roller bearings," Zeitschrift Des Vereines Deutcher Ingenieure, vol. 46, no. 36-38, pp. 1342-1348, 1432-1437, 1902.

[22] L. Ljung, System Identification: Theory for the User (2nd Edition). Prentice Hall PTR, December 1998.

[23] C. J. Seeton, "Viscosity-temperature correlation for liquids," Tribology Letters, vol. 22, no. 1, pp. 67-78, Mar. 2006. 